https://doi.org/10.15407/ujpe66.4.281

Y.A. ABBO

Wollega University, Department of Physics

(P.O. Box 359, Nekemte, Ethiopia)

\title{
SLOW AND FAST LIGHTS \\ IN METAL/DIELECTRIC COMPOSITE \\ OF CYLINDRICAL NANOINCLUSIONS \\ IN PASSIVE AND ACTIVE LINEAR \\ DIELECTRIC HOST MATRICES
}

\begin{abstract}
This paper presents theoretical discussions and computational numerical results obtained from the study of extreme values of the speed of light in metal/dielectric composite, where the cylindrical nanoinclusions are uniformly distributed in the linear dielectric host matrix. The results testify that, within our approach, at the region of anomalous dispersion, light can travel with a group velocity greater than the speed of light in vacuum. In a composite with passive host matrix, the light pulse is absorbed within a very small distance. The problem of absorption can be reduced considerably by using an active host matrix.

Ke y words: slow light, fast light, nanocomposite, group velocity.
\end{abstract}

\section{Introduction}

Early investigations of slow and superluminal light have been conducted in atomic media at a cryogenic temperature [1]. Hau et al. [2] showed slow light in an ultracold sodium vapor. This report was followed by that of Kash et al. [3], who found that the ultraslow light speed in a hot atomic vapor of rubidium. Akulashin et al. [4] and Kim et al. [5] observed fast light using the method of electromagnetically induced absorption, and Wang et al., [6] also demonstrated fast light using the gain-assisted superluminal light propagation which was first introduced by Steinberg and Chiao [7].

The investigation of slow and fast light in solidstate materials recently becomes an active promising area of studies due to its application to quantum electronics, high-performance communication, controllable optical delay lines, optical data storage, and optical memories.

(C) Y.A. ABBO, 2021

ISSN 2071-0194. Ukr. J. Phys. 2021. Vol. 66, No. 4
Recently, the study of slow and superluminal light focuses on solid-state materials at room temperature (see, e.g., Bigelow et al. [8]). V.N. Mal'nev and Sisay [9] theoretically investigated slow, superluminal, and backward lights in a composite of spherical nanoinclusions in active/passive host matrices. Kim and Choe [10] studied slow and stopped lights in a composite with spherical metallic nanoinclusions.

This paper presents the computational results obtained from the study of the speed of light in a nanocomposite with cylindrical nanoinclusions embedded in a linear dielectric host matrix. We will show that the shape of nanoinclusions is of significance for the extreme values of the speed of light in a composite with spherical nanoinclusions and in a composite with cylindrical nanoinclusions. To the author's knowledge, the study of slow, fast, and backward lights in a nanocomposite with cylindrical nanoinclusions is first presented in this work. The obtained results are original. 


\section{Electric Potential Distribution}

In the cylindrical coordinate system, the Laplace equation (i.e., $\nabla^{2} \Phi=0$ ) takes the form [11]

$\frac{\partial^{2} \Phi}{\partial r^{2}}+\frac{1}{r} \frac{\partial \Phi}{\partial r}+\frac{1}{r^{2}} \frac{\partial^{2} \Phi}{\partial \theta^{2}}+\frac{\partial^{2} \Phi}{\partial z^{2}}=0$.

Since the potential is not a function of the $z$ coordinate, Eq. (1) can be reduced to the form

$\frac{\partial^{2} \Phi}{\partial r^{2}}+\frac{1}{r} \frac{\partial \Phi}{\partial r}+\frac{1}{r^{2}} \frac{\partial^{2} \Phi}{\partial \theta^{2}}=0$.

Consider the composite of a metal covered by dielectric cylindrical nanoinclusions uniformly embedded in a dielectric host matrix. The electrical potential distribution at different regions of the composite can be determined from the solution of the Laplace equation given by Eq. (2) as [12]

$\Phi_{1}=-\mathbf{E}_{h} A r \cos \theta, \quad r \leq r_{1}$,

$\Phi_{2}=-\mathbf{E}_{h}\left(B r-\frac{C}{r}\right) \cos \theta, \quad r_{1} \leq r \leq r_{2}$,

$\Phi_{3}=-\mathbf{E}_{h}\left(r-\frac{D}{r}\right) \cos \theta, \quad r \geq r_{2}$,

where $\Phi_{1}, \Phi_{2}$, and $\Phi_{3}$ are potentials in the dielectric core, metallic shell, and the dielectric host matrix, respectively, $\mathbf{E}_{h}$ is the electric field in the host, $r_{1}$ and $r_{2}$ are the radii of the dielectric core and the metal shell, respectively, and, $A, B, C$, and $D$ are unknown coefficients which can be obtained from electrostatics boundary conditions.

The unknown coefficients $A, B, C$, and $D$ can be obtained from electrostatic boundary conditions in the long-wave approximation, the case in which the wavelength of an incident electromagnetic wave is greater than the size of a particle (i.e., $r_{2} \ll \lambda$ ). The expressions for the unknown constants in terms of the dielectric constant of the host $\left(\epsilon_{h}\right)$, metal cover $\left(\epsilon_{2}\right)$, dielectric core $\left(\epsilon_{1}\right)$, and metal volume fraction in the inclusion $(p)$ have the form

$$
\begin{aligned}
A & =\frac{4 \epsilon_{2} \epsilon_{h}}{p \Delta}, \\
B & =\frac{2 \epsilon_{h}\left(\epsilon_{1}+\epsilon_{2}\right)}{p \Delta}, \\
C & =\frac{2 \epsilon_{h}\left(\epsilon_{1}-\epsilon_{2}\right) r_{1}^{2}}{p \Delta}, \\
D & =\left[1-2 \epsilon_{h} \frac{\left[\epsilon_{2}(2-p)+\epsilon_{1} p\right]}{p \Delta}\right] r_{2}^{2},
\end{aligned}
$$

where

$p=1-\left(\frac{r_{1}}{r_{2}}\right)^{2}$,

$\Delta=\epsilon_{2}^{2}+q \epsilon_{2}+\epsilon_{1} \epsilon_{h}$,

$q=\left(\frac{2}{p}-1\right) \epsilon_{1}+\left(\frac{2}{p}-1\right) \epsilon_{h}$.

\section{Effective Polarizability}

The effective polarizability of an individual metalcovered dielectric cylindrical nanoinclusions embedded in a dielectric host matrix can be presented by referring Eqs. (5) and (9) as [12]

$D=\beta r_{2}^{2}$,

where

$\beta=1-2 \frac{\delta}{\Delta}$,

$\delta=\epsilon_{h}\left[\epsilon_{2}\left(\frac{2}{p}-1\right)+\epsilon_{1}\right]$,

$\Delta=\epsilon_{2}^{2}+q \epsilon_{2}+\epsilon_{1} \epsilon_{h}$

$p=1-\left(\frac{r_{1}}{r_{2}}\right)^{2}$,

$q=\left(\frac{2}{p}-1\right) \epsilon_{1}+\left(\frac{2}{p}-1\right) \epsilon_{h}$.

The dielectric function of the core $\epsilon_{1}$ is chosen to be real and frequency-independent. The dielectric constant of the host medium $\epsilon_{h}$ is a complex value, and the dielectric function of the metal cover $\epsilon_{2}$ is chosen to have the Drude form

$\epsilon_{2}=\epsilon_{\infty}-\frac{1}{z(z+i \gamma)}$,

with,

$\epsilon_{2}^{\prime}=\varepsilon_{\infty}-\frac{1}{z^{2}+\gamma^{2}}$,

$\epsilon_{2}^{\prime \prime}=\frac{\gamma}{z\left(z^{2}+\gamma^{2}\right)}$,

where $\epsilon_{2}^{\prime}$ and $\epsilon_{2}^{\prime \prime}$ are the real and imaginary parts of $\epsilon_{2}$, respectively, $z=\frac{\omega}{\omega_{p}}$ is the dimensionless frequency, $\omega$ is the incident radiation frequency, $\omega_{p}$ is the plasma frequency of the metal part, $\gamma=\frac{\nu}{\omega_{p}}$, and $\nu$ is the electron collision frequency.

ISSN 2071-0194. Ukr. J. Phys. 2021. Vol. 66, No. 4 
Since $\epsilon_{2}$ and $\epsilon_{h}$ are complex, the terms such as $q$, $\delta, \Delta$, and $\beta$ are also complex:

$\Delta=\Delta^{\prime}+i \Delta^{\prime \prime}$,

$\delta=\delta^{\prime}+i \delta^{\prime \prime}$,

$\beta=\beta^{\prime}+i \beta^{\prime \prime}$,

$q=q^{\prime}+i q^{\prime \prime}$

$\epsilon_{h}=\epsilon_{h}^{\prime}+i \epsilon_{h}^{\prime \prime}$

where

$\Delta^{\prime}=\left(\epsilon_{2}^{\prime 2}-\epsilon_{2}^{\prime \prime 2}+q^{\prime} \epsilon_{2}^{\prime}-q^{\prime \prime} \epsilon_{2}^{\prime \prime}+\epsilon_{1} \epsilon_{h}^{\prime}\right)$,

$\Delta^{\prime \prime}=\left(2 \epsilon_{2}^{\prime} \epsilon_{2}^{\prime \prime}+q^{\prime} \epsilon_{2}^{\prime \prime}+q^{\prime \prime} \epsilon_{2}^{\prime}+\epsilon_{1} \epsilon_{h}^{\prime \prime}\right)$,

$\delta^{\prime}=\left(\frac{2}{p}-1\right)\left[\epsilon_{2}^{\prime} \epsilon_{h}^{\prime}-\epsilon_{2}^{\prime \prime} \epsilon_{h}^{\prime \prime}\right]+\epsilon_{1} \epsilon_{h}^{\prime}$,

$\delta^{\prime \prime}=\left(\frac{2}{p}-1\right)\left[\epsilon_{2}^{\prime} \epsilon_{h}^{\prime \prime}+\epsilon_{2}^{\prime \prime} \epsilon_{h}^{\prime}\right]+\epsilon_{1} \epsilon_{h}^{\prime \prime}$,

$\beta^{\prime}=1-2 \frac{\delta^{\prime} \Delta^{\prime}+\delta^{\prime \prime} \Delta^{\prime \prime}}{\Delta^{\prime 2}+\Delta^{\prime \prime 2}}$,

$\beta^{\prime \prime}=2 \frac{\delta^{\prime} \Delta^{\prime \prime}-\delta^{\prime \prime} \Delta^{\prime}}{\Delta^{\prime 2}+\Delta^{\prime \prime 2}}$

$q^{\prime}=\left(\frac{2}{p}-1\right) \epsilon_{1}+\left(\frac{2}{p}-1\right) \epsilon_{h}^{\prime}$

$q^{\prime \prime}=\left(\frac{2}{p}-1\right) \epsilon_{h}^{\prime \prime}$.

For a composite with pure metal cylindrical inclusions, the corresponding quantities for $\beta^{\prime}$ and $\beta^{\prime \prime}$ are given, respectively, by

$\beta_{m}^{\prime}=\frac{\left(\epsilon_{2}^{\prime}-\epsilon_{h}^{\prime}\right) \Delta_{m}^{\prime}+\left(\epsilon_{2}^{\prime \prime}-\epsilon_{h}^{\prime \prime}\right) \Delta_{m}^{\prime \prime}}{\left|\Delta_{m}\right|^{2}}$,

$\beta_{m}^{\prime \prime}=\frac{\left(\epsilon_{2}^{\prime \prime}-\epsilon_{h}^{\prime \prime}\right) \Delta_{m}^{\prime}+\left(\epsilon_{2}^{\prime}-\epsilon_{h}^{\prime}\right) \Delta_{m}^{\prime \prime}}{\left|\Delta_{m}\right|^{2}}$,

where

$\left|\Delta_{m}\right|^{2}=\Delta_{m}^{\prime 2}+\Delta_{m}^{\prime \prime 2}$,

$\Delta_{m}^{\prime}=\epsilon_{2}^{\prime}+\epsilon_{h}^{\prime}$

$\Delta_{m}^{\prime \prime}=\epsilon_{2}^{\prime \prime}+\epsilon_{h}^{\prime \prime}$.

ISSN 2071-0194. Ukr. J. Phys. 2021. Vol. 66, No. 4

\section{Refractive Index}

According to the effective medium theory, for the study of the optical properties of a composite with an inhomogeneity scale much smaller than the wavelength of interest, the electrodynamic quantities of each of the constituent particles are overshadowed by the average response of the whole system. Therefore, the optical properties of a microscopically heterogeneous composite should be investigated by evaluating the effective dielectric function in terms of the permittivities of individual components, as well as their respective volume fractions [13].

The explicit form of the Rayleigh rule for a composite with cylindrical inclusions, which is known as the Maxwell-Garnet formula, can be written as [14]

$\epsilon_{f f}=\epsilon_{h}\left(1+\frac{2 f \beta}{1-f \beta}\right)$

where $\beta=\frac{\epsilon_{i}-\epsilon_{h}}{\epsilon_{i}+\epsilon_{h}}, \epsilon_{f f}$ is the effective permittivity of the composite, $f$, the volume fraction of inclusions in the composite, and $\epsilon_{i}$ is the dielectric constant of the $i^{\text {th }}$ inclusion. For a nonmagnetic medium, $\mu=\mu_{0}$. Hence, the index of refraction $n$ can be expressed in terms of the effective dielectric constant as $n^{2}=\epsilon_{f f}$. Therefore, it is possible to determine the refractive index of the composite from the above formula as

$n^{2}=\epsilon_{h}\left(1+\frac{2 f \beta}{1-f \beta}\right)$.

Quantities such as $\epsilon_{h}$ and $\beta$ are complex, so that it is important to express $n^{2}$ in terms of its real and imaginary parts:

$n^{2}=\left(\epsilon_{h}^{\prime}+i \epsilon_{h}^{\prime \prime}\right)\left[1+2 f \frac{\left(\beta^{\prime}+i \beta^{\prime \prime}\right)}{\left(1-f \beta^{\prime}\right)-i f \beta^{\prime \prime}}\right]=b_{1}+i b_{2}$,

where

$b_{1}=\left(\epsilon_{h}^{\prime}+2 f\left[\frac{\left(\beta^{\prime}-f|\beta|^{2}\right) \epsilon_{h}^{\prime}-\beta^{\prime \prime} \epsilon_{h}^{\prime \prime}}{\Delta_{f}}\right]\right)$,

$b_{2}=\left(\epsilon_{h}^{\prime \prime}+2 f\left[\frac{\left(\beta^{\prime}-f|\beta|^{2}\right) \epsilon_{h}^{\prime \prime}+\beta^{\prime \prime} \epsilon_{h}^{\prime}}{\Delta_{f}}\right]\right)$,

$\Delta_{f}=1-f \beta^{\prime}+f^{2}|\beta|^{2}$.

Equating the real and the imaginary parts $n=n^{\prime}+$ $+i n^{\prime \prime}$ with Eq. (21) can give us the expressions

$n^{\prime 2}-n^{\prime \prime 2}=b_{1}$,

$2 n^{\prime} n^{\prime \prime}=b_{2}$. 

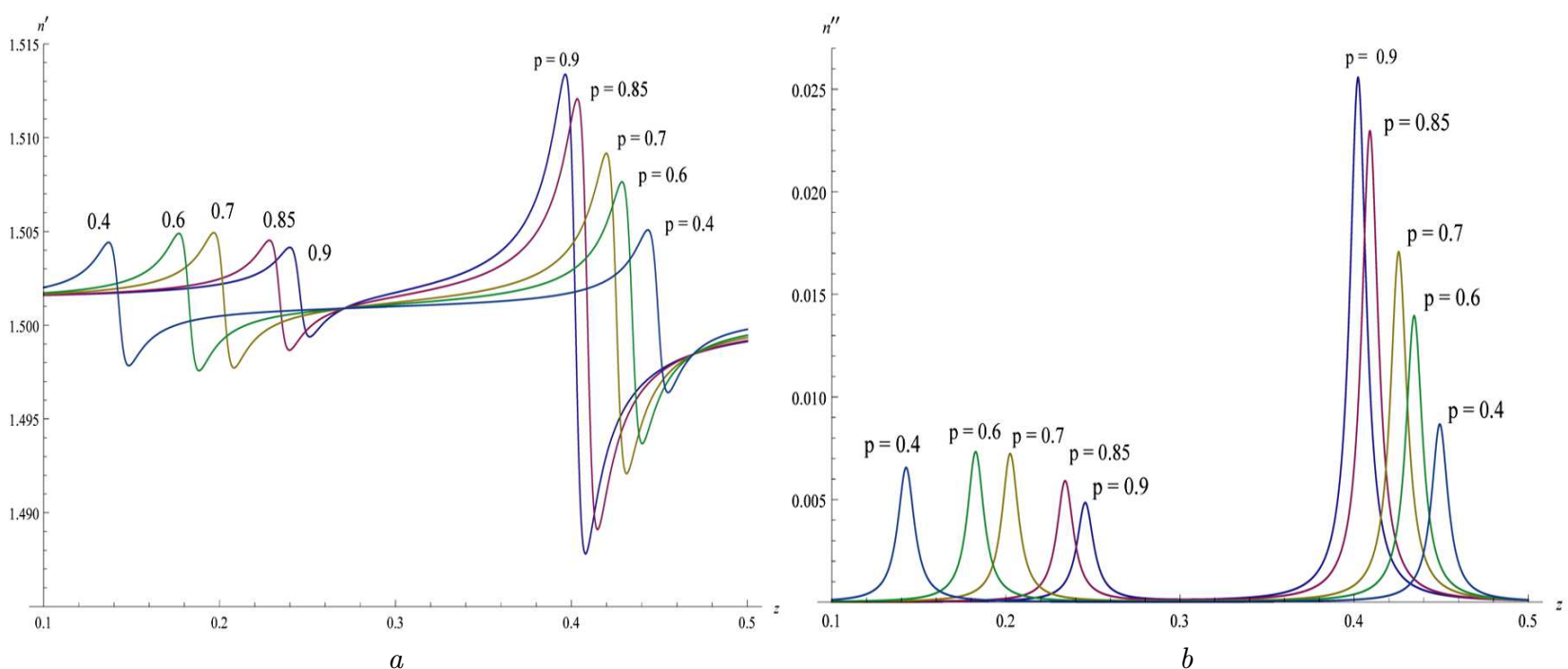

Fig. 1. Passive composite with tuned cylindrical nanoinclusions $\left(\epsilon_{h}^{\prime \prime}=0\right)$. Real part of the refractive index $n^{\prime}$ versus the dimensionless frequency $z(a)$; imaginary part of the refractive index $n^{\prime \prime}$ versus $z(b)$. Numerical values of composite parameters; metal fraction in the inclusion $p=0.4,0.6,0.7,0.85$, and 0.9 , fraction of inclusions in the composite $f=0.001$

The real and imaginary parts of the refractive index can be written in a condensed form by using $b_{1}$ and $b_{2}$ as

$n^{\prime 2}=\frac{1}{2}\left(\sqrt{b_{1}^{2}+b_{2}^{2}}+b_{1}\right)$,

$n^{\prime \prime 2}=\frac{1}{2}\left(\sqrt{b_{1}^{2}+b_{2}^{2}}-b_{1}\right)$.

Figure $1, a$ shows $n^{\prime}$ versus $z$. Figure $1, b$ gives $n^{\prime \prime}$ versus $z$ near and at resonant frequencies of the composite of a metal covered by dielectric cylindrical inclusions in the passive host matrix for different metal fractions $p$. For a composite with coated inclusions, the obtained results show that there are two peak values of $n^{\prime}$ and $n^{\prime \prime}$ at two different resonant frequencies. The left peak is smaller in magnitude than the one on the right side for both $n^{\prime}$ and $n^{\prime \prime}$. The large

Constant values used in numerical calculations

\begin{tabular}{|c|c|}
\hline Constants & Values \\
\hline$\epsilon_{0}$ & 4.5 \\
$\epsilon_{h}^{\prime}$ & 2.25 \\
$\epsilon_{1}=4 \epsilon_{h}^{\prime}$ & 6 \\
$\omega_{p}$ (silver $)$ & $1.45 \times 10^{16}$ \\
$\nu$ & $1.68 \times 10^{14}$ \\
$\gamma$ & $1.15 \times 10^{-2}$ \\
\hline
\end{tabular}

positive value of the imaginary part $n^{\prime \prime}$ of the refractive index implies that, at the two resonant frequencies, there is the strong absorption of an electromagnetic wave that propagates through the composite.

Unlike the coated inclusion case, we observe in Fig. 2, $a$ and Fig. 2, $b$ that, for a composite with pure metal cylindrical inclusions, there is only one peak value of $n^{\prime}$ and $n^{\prime \prime}$. From the plots, we can see, varying the fraction of inclusions $f$ in the composite, a change in the magnitude of the peak value. Again in a composite with pure metal cylindrical inclusions in the passive host matrix, there is the strong absorption of an incident electromagnetic wave at the region of anomalous dispersion. The wave equation admits a solution in the form of a plane wave $E=E_{0} e^{i(k x-\omega t)}$. The wave number $k=\frac{n \omega}{c}$ is a complex quantity and has the form $k=k^{\prime}+i k^{\prime \prime}$. So we can write the above equation as [15]:

$\mathbf{E}=\mathbf{E}_{0} e^{i\left(k^{\prime} x-\omega t\right)} e^{-k^{\prime \prime} x}$

The presence of the term $e^{-k^{\prime \prime} x}$ implies that the wave decays, as it propagates. The intensity of an electromagnetic wave is proportional to the square of the electric field (i.e., $I \sim \mathbf{E}^{2}$ ). From Beer's law, we have the relation

$I=I_{0} e^{-\alpha_{\text {abso }} x}$, 

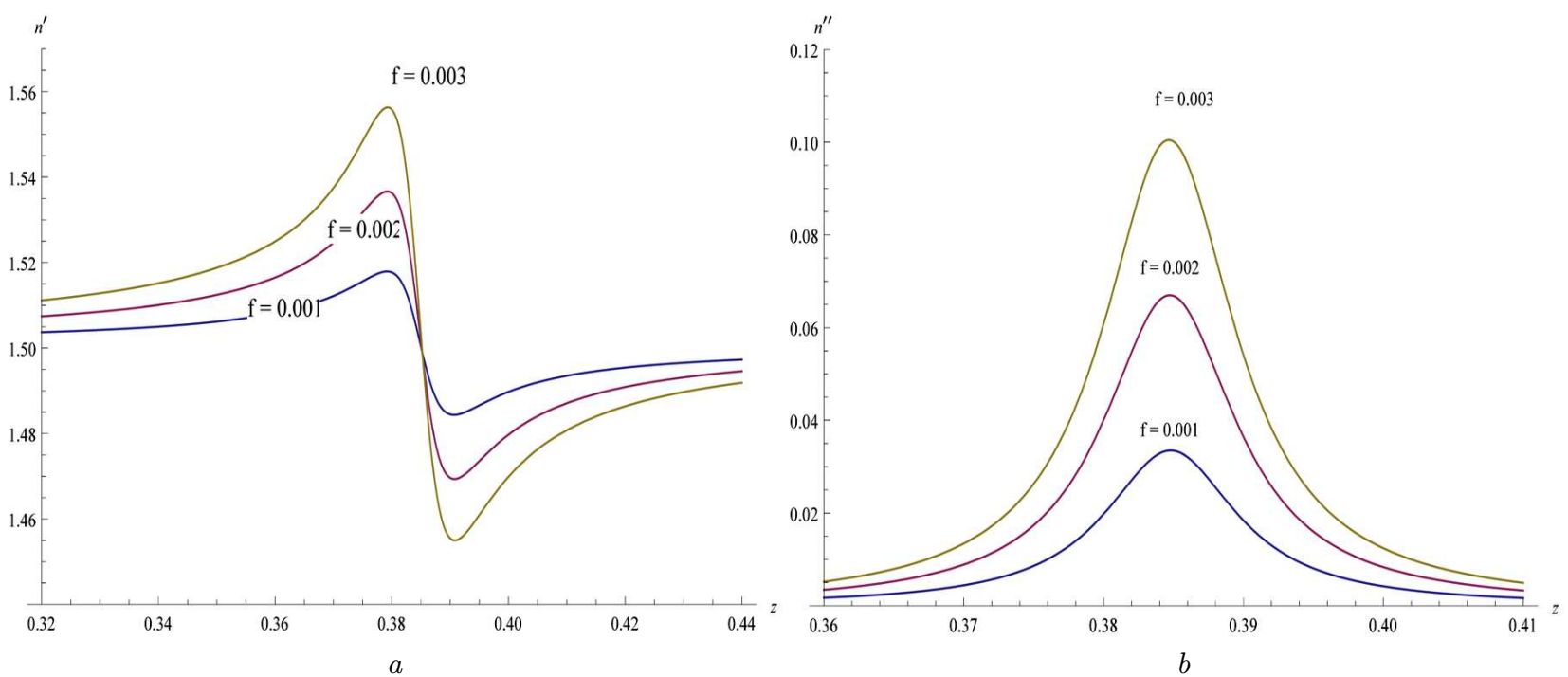

Fig. 2. Passive composite with pure metal cylindrical nanoinclusions $\left(\epsilon_{h}^{\prime \prime}=0\right)$. Real part of the refractive index $n^{\prime}$ versus the dimensionless frequency $z(a)$; imaginary part of the refractive index $n^{\prime \prime}$ versus $z(b)$. Numerical values of composite parameters; fractions of inclusions in the composite $f=0.001,0.002$, and 0.003

where $\alpha_{\text {abso }}$ is known as the absorption coefficient. Therefore, we can write the absorption coefficient as

$\alpha_{\text {abso }}=2 k^{\prime \prime}=2 n^{\prime \prime} \omega / c$,

$\alpha_{\text {abso }}=2 n^{\prime \prime} z_{r} \omega_{p} / c$.

For a composite of tuned inclusions with metal fraction $p=0.9$ in a passive host matrix, $n^{\prime \prime}=0.025$ at the resonant frequency $z_{r}=0.4$. Using $\omega_{p}=$ $=1.6 \times 10^{16}$, we get that $\alpha_{\text {abso }}=1 \times 10^{4} \mathrm{~cm}^{-1}$. The typical light propagation length in the media can be found with the help of $l \sim 1 / \alpha_{\text {abso }}$ [16]. So, in our case, $l \approx 9.4 \times 10^{-5} \mathrm{~cm}$.

For a composite of pure metal inclusions in the passive host matrix, we got that $n^{\prime \prime}=0.033$ at the resonant frequency $z_{r}=0.385$. Using $\omega_{p}=1.6 \times 10^{16}$ we obtain $\alpha_{\text {abso }}=1.4 \times 10^{4} \mathrm{~cm}^{-1}$. In our case, $l \approx 7.4 \times 10^{-5} \mathrm{~cm}$.

In order to create the condition for considerably propagating light waves, it is necessary to decrease $n^{\prime \prime}$. This can be done by introducing a negative part into the dielectric function of the host matrix [5]. The host matrix with negative $\epsilon_{h}$ (i.e., $\epsilon_{h}^{\prime \prime}<0$ ) amplifies the incident electromagnetic wave rather than absorbs it, such medium is known as the active host matrix.

Figures 3, $a$ and $b$ show, respectively, the real and imaginary parts of a composite of tuned inclusions in the active host matrix for different metal fractions $p$. In Fig. 3, $a$, we can see two maximum values of the real part of the refractive index at two different resonant frequencies. The first peak of the real part of the refractive index, on the left side, is comparatively very small relative to the second peak on the right. In Fig. $3, b$, for $p=0.85$, the imaginary part of the refractive index has two minimum values at two different resonant frequencies. For the metal thicknesses $p=0.9$ and $p=0.95$, the imaginary part of the refractive index has a fork like the structure at the second resonant frequency on the right side. For a composite of tuned inclusions with the metal fraction $p=0.9$ in the active host matrix (i.e., $\epsilon_{h}=-0.13866$ ), $n^{\prime \prime}=0.0001361$ at the resonant frequency $z_{r}=0.4$ for $\omega_{p}=1.6 \times$ $\times 10^{16}$, we get that $\alpha_{\text {abso }}=48.4736 \mathrm{~cm}^{-1}$. The typical light propagation length in the media can be found with the help of $l \sim 1 / \alpha_{\text {abso }}$ [16]. In our case, $l \approx 0.02 \mathrm{~cm}$.

The real and imaginary parts of the refractive index for a composite with pure metal inclusions in the active host matrix for different fractions of inclusions (i.e., $f$ ) presented in Fig. 4, $a$ and Fig. 4, b, respectively. Figure $4, a$ for different $f$ shows that there is only one maximum of $n^{\prime}$, and its magnitude can be increased by increasing $f$. The imaginary part $n^{\prime \prime}$ for $f=0.0001$ and $f=0.0002$ has 

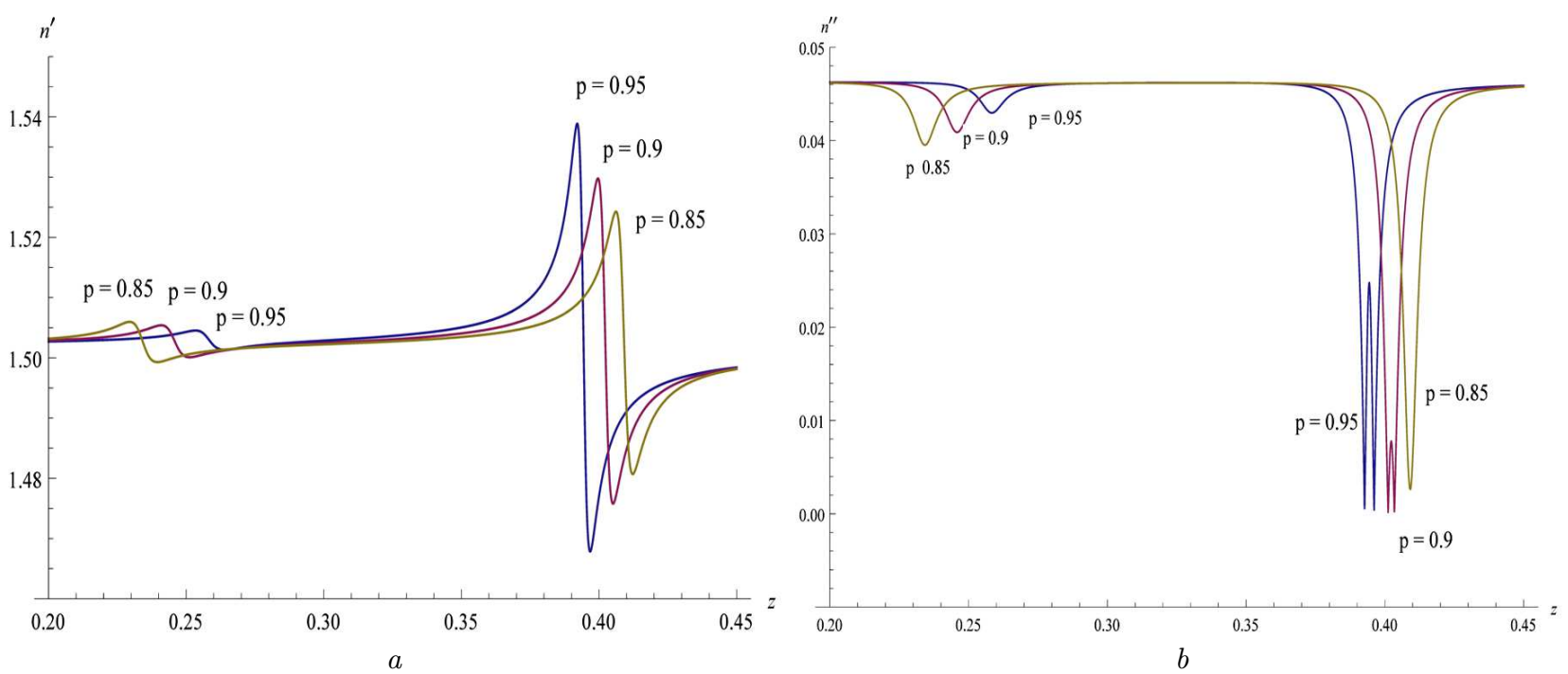

Fig. 3. Active composite with tuned cylindrical nanoinclusions $\left(\epsilon_{h}^{\prime \prime}=-0.13866\right)$. Real part of the refractive index $n^{\prime}$ versus the dimensionless frequency $z(a)$; imaginary part of the refractive index $n^{\prime \prime}$ versus $z(b)$. Numerical values of the composite parameters: the metal fractions in inclusions $p=0.85,0.9$, and 0.95 , fraction of inclusions in the composite $f=0.001$
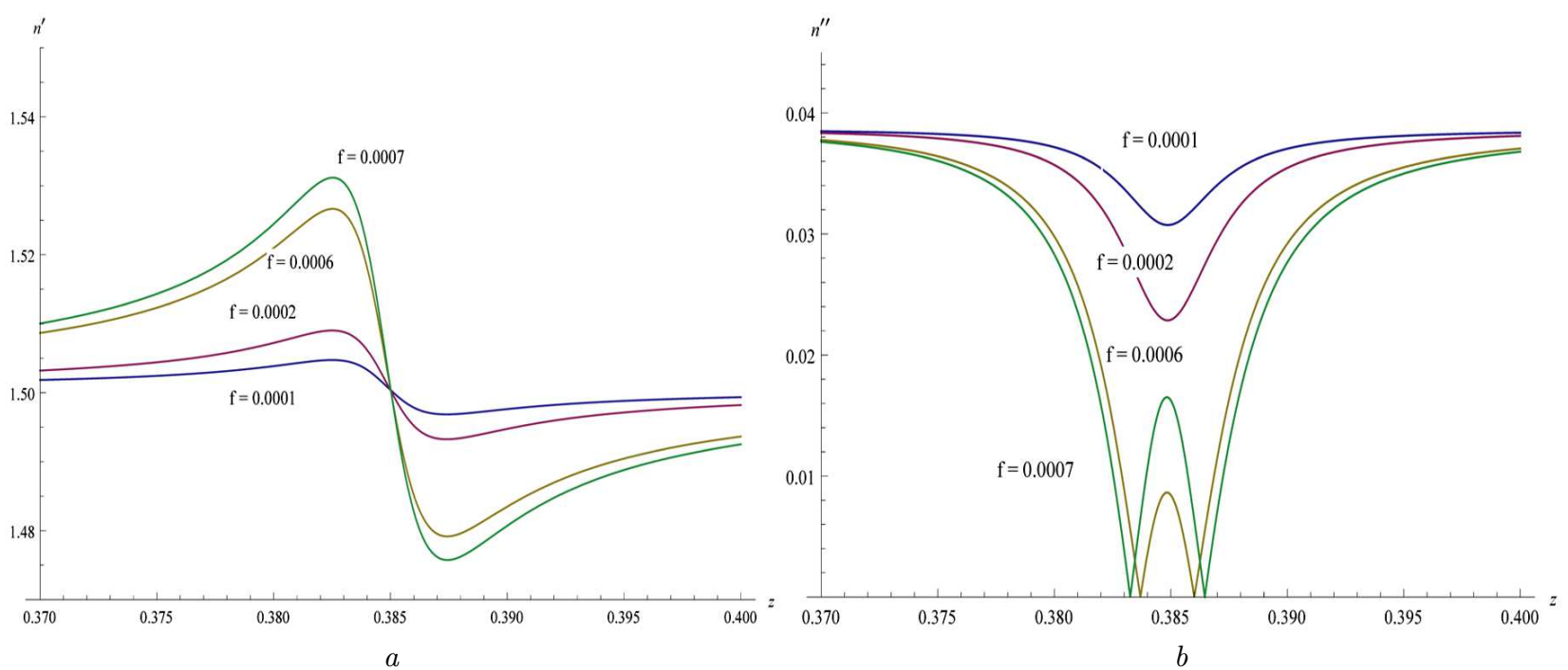

Fig. 4. Active composite with pure metal cylindrical nanoinclusions $\left(\epsilon_{h}^{\prime \prime}=-0.1159113\right)$. Real part of the refractive index $n^{\prime}$ versus the dimensionless frequency $z(a)$; imaginary part of the refractive index $n^{\prime \prime}$ versus $z(b)$. Numerical values of the composite parameters: the fractions of inclusions in the composite $f=0.0001,0.0002,0.0006$, and 0.0007

only one minimum. When we increase the fraction of inclusions to $f=0.0006$ and $f=0.0007$, we get that the minimum value has a fork-like structure. The minimum value of $n^{\prime \prime}$ becomes lower, as we increase $f$.

For pure metal inclusions in active host matrices, we have found that the value of $n^{\prime \prime}$ is significantly reduced, and it becomes $n^{\prime \prime}=5.9 \times 10^{-5}$. The typical length of light propagating in a composite of pure metal cylindrical inclusions becomes $l \approx 0.04 \mathrm{~cm}$.

\section{Slow, Superluminal, and Backward Lights}

The notion of group velocity is related to a wave packet (pulse), and it can be defined as the velocity with the overall shape of the wave's amplitude 
known as the modulation or the envelope of a wave propagating through space [11].

A narrow wave packet can be represented by

$U(x, t)=e^{i\left(k_{0} x-\omega_{0} t\right)} \int_{k_{0}-\delta k}^{k_{0}+\delta k} A(k) e^{i\left(k x-\frac{d \omega}{d k} \mid k_{0} t\right)\left(k-k_{0}\right)}$.

This shows that the traveling pulse is undistorted in shape and has a velocity called the group velocity $\left(V_{g}\right)$ given by

$V_{g}=\frac{d \omega}{d k} \mid k_{0}$.

The group velocity can be expressed in terms of the group refractive index as

$V_{g}=\frac{c}{n^{\prime}(\omega)+\omega \frac{d n^{\prime}(\omega)}{d \omega}}$,

where $c$ is the speed of light in vacuum. In order to discuss the concepts of slow, fast, and negative group velocities of light, we use the expression for the group velocity in terms of the group refractive index

$\mathbf{v}_{g}=\frac{c}{n^{\prime}(\omega)+\omega \frac{d n^{\prime}(\omega)}{d \omega}}=\frac{c}{n_{g}}$

where $n^{\prime}(\omega)+\omega \frac{d n^{\prime}(\omega)}{d \omega}$ is the group index.

We can see that the second term (i.e., $\omega \frac{d n}{d \omega}$ ) plays a great role in obtaining the extreme values of group velocity. Slow light refers to the situation in which $\mathbf{v}_{g} \ll c$. For normal dispersion $\frac{d n}{d \omega}>0$, and $n>1$; then the group velocity is less than the phase velocity. Fast light refers to the light traveling faster than the speed of light in vacuum. This circumstance can occur either when $\mathbf{v}_{g}>c$ or when the group velocity is negative and large. In a region of anomalous dispersion, $\frac{d n}{d \omega}$ can become large and negative. Then the group velocity differs greatly from the phase velocity, often becoming larger than $c$ or even negative [2].

According to the special theory of relativity, nothing can travel with speed faster than the speed of light in vacuum. The concept of superluminal group velocity doesn't violate the special theory of relativity, because, at the region of anomalous dispersion, the group velocity doesn't have physical meaning. Large $\frac{d n}{d \omega}$ implies the existence of a significant absorption and a rapid variation of $\omega$ with $k$. Consequently, the approximation made on the driving group velocity are no longer valid. Usually, a pulse with its dominant frequency components in a neighborhood of the strong absorption line is absorbed and distorted, as it travels [11].

Garret and McCumber [17] showed that there are circumstances in which the superluminal group velocity can still have meaning even with anomalous dispersion. S. Chu and S. Wong subsequently verified experimentally what Garret and McCumber showed theoretically: namely, if absorbers are not too thick and for a narrow band light pulse [18].

A negative group velocity corresponds to the case where the peak of the pulse transmitted through an optical material emerges before the peak of the incident light field enters the medium. One might fear that the existence of a negative group velocity would lead to a violation of the notion of causality. But physically, what occurs is the pulse reshaping. Any physical pulse will have leading and trailing wings, the leading wing contains the information about the entire pulse shape, and this information traveling at normal velocities such as the speed of light in vacuum will allow the output pulse to be fully reconstructed long before the peak of the input pulse enters the medium [14].

The numerical values of the group velocity near and at the resonant frequency can be determined by using Eq. (35) with the real part of the refractive index as a function of the dimensionless frequency $z$. All the values of the parameters used to find the group velocity are the same as those used in finding the real and imaginary parts of the refractive index. We have

$\mathbf{v}_{g}=\frac{c}{n^{\prime}(z)+z \frac{d n^{\prime}(z)}{d z}}=\frac{c}{n_{g}(z)}$.

\section{Results and Discussion}

\subsection{Passive host matrix}

Figures 5-7 show the group refractive index and the group velocity for tuned cylindrical inclusions in the passive host matrix with $p=0.9, p=0.7$, and $p=$ $=0.4$, respectively.

From the obtained numerical results, (Fig. 5, b), we see that, for $p=0.9$ at the region of the first resonant frequency, the group velocity of slow light $\mathbf{v}_{g}=0.77 c$. In the region of the second resonant frequency, there are two singularity points. When the 

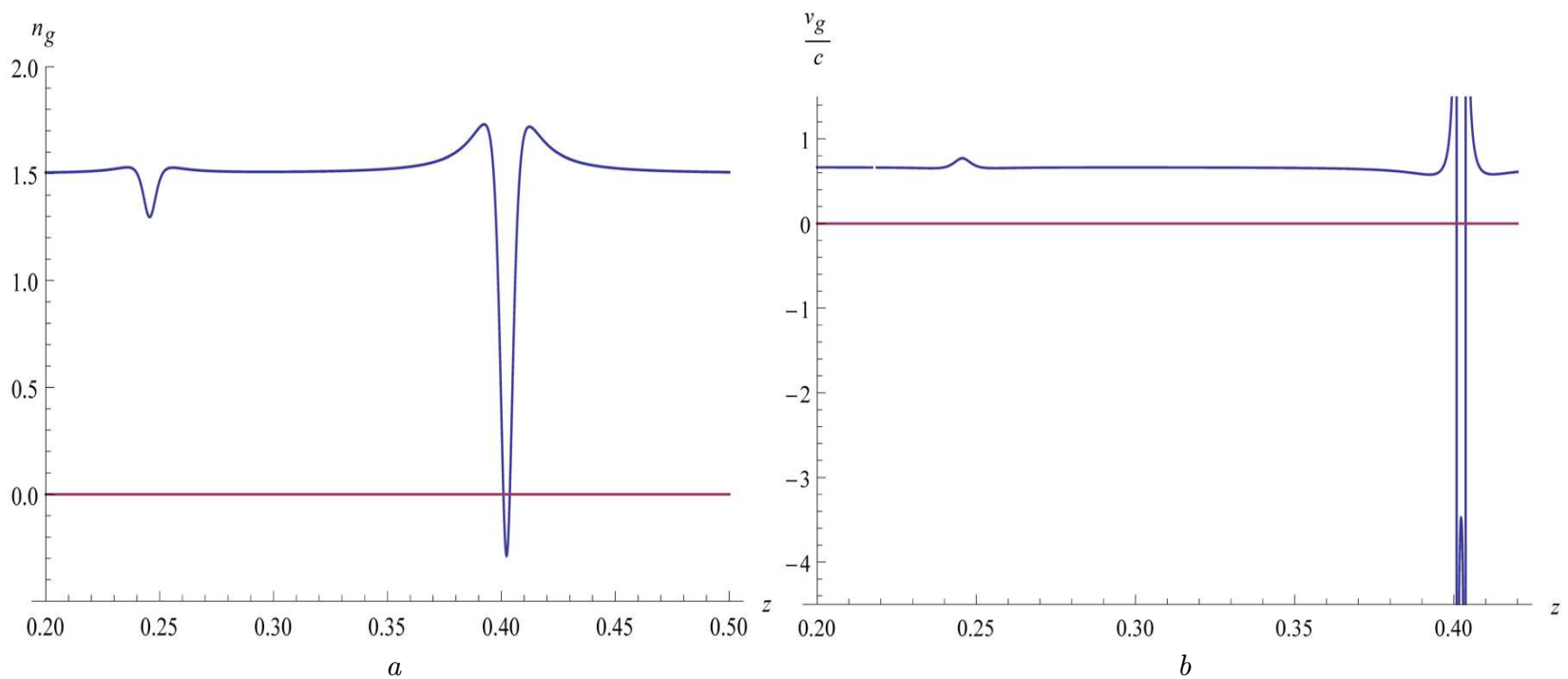

Fig. 5. Passive composite with tuned cylindrical nanoinclusions $\left(\epsilon_{h}^{\prime \prime}=0\right)$. Its group index $n_{g}$ versus the dimensionless frequency $z(a)$. The normalized group velocity $\mathbf{v}_{g} / c$ versus $z(b)$. Numerical values of composite parameters: metal fraction in an inclusion $p=0.9$, fraction of inclusions in the composite $f=0.001$
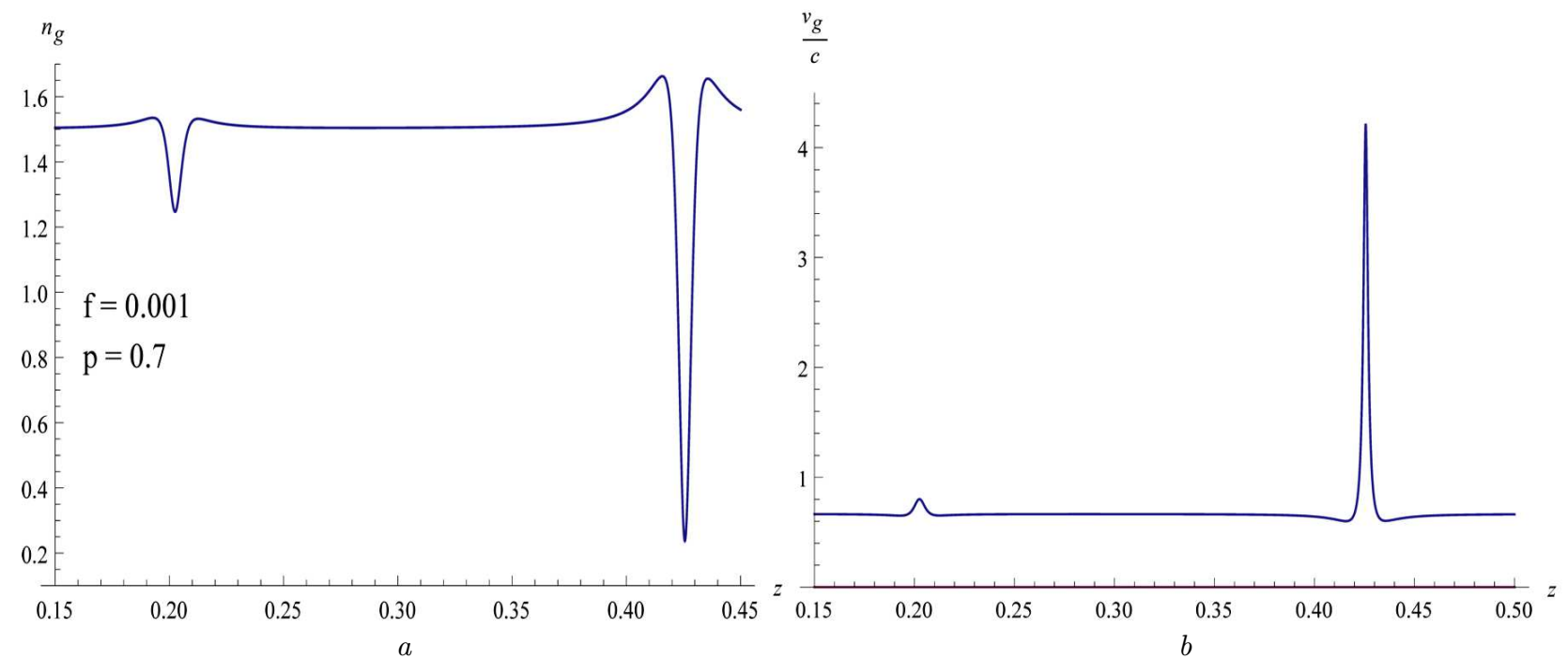

Fig. 6. Passive composite with tuned cylindrical nanoinclusions $\left(\epsilon_{h}^{\prime \prime}=0\right)$. Its group index $n_{g}$ versus the dimensionless frequency $z(a)$. The normalized group velocity $\mathbf{v}_{g} / c$ versus $z(b)$. Numerical values of composite parameters: metal fraction in the inclusion $p=0.7$, fraction of inclusions in the composite $f=0.001$

frequency approaches from the left to the first asymptote line, as well as to the second asymptote line from the right, $\mathbf{v}_{g} / c \rightarrow+\infty$. Between the two asymptote lines on the right side, we get a negative superluminal light of group velocity $\mathbf{v}_{g}=-3.5 c$.

Reducing the thickness of a metal in the inclusions shows a significant change in the result, and we found two maxima of the normalized group velocity.
For $p=7$ and $p=0.4$, the plots for $\mathbf{v}_{g} / c$ versus $z$ in Fig. 6, $b$ and Fig. 7, $b$, respectively, depict the identical type of curves. For both $p=0.7$ and $p=0.4$, the first peak on the left side shows of the slow-light group velocities $\mathbf{v}_{g}=0.8 c$ and $\mathbf{v}_{g}=0.75 c$, respectively. Unlike the left peak, the right peak gives the superluminal-light group velocities $\mathbf{v}_{g}=4 c$ and $\mathbf{v}_{g}=1.2 c$ for $p=0.7$ and $p=0.4$, respectively. 

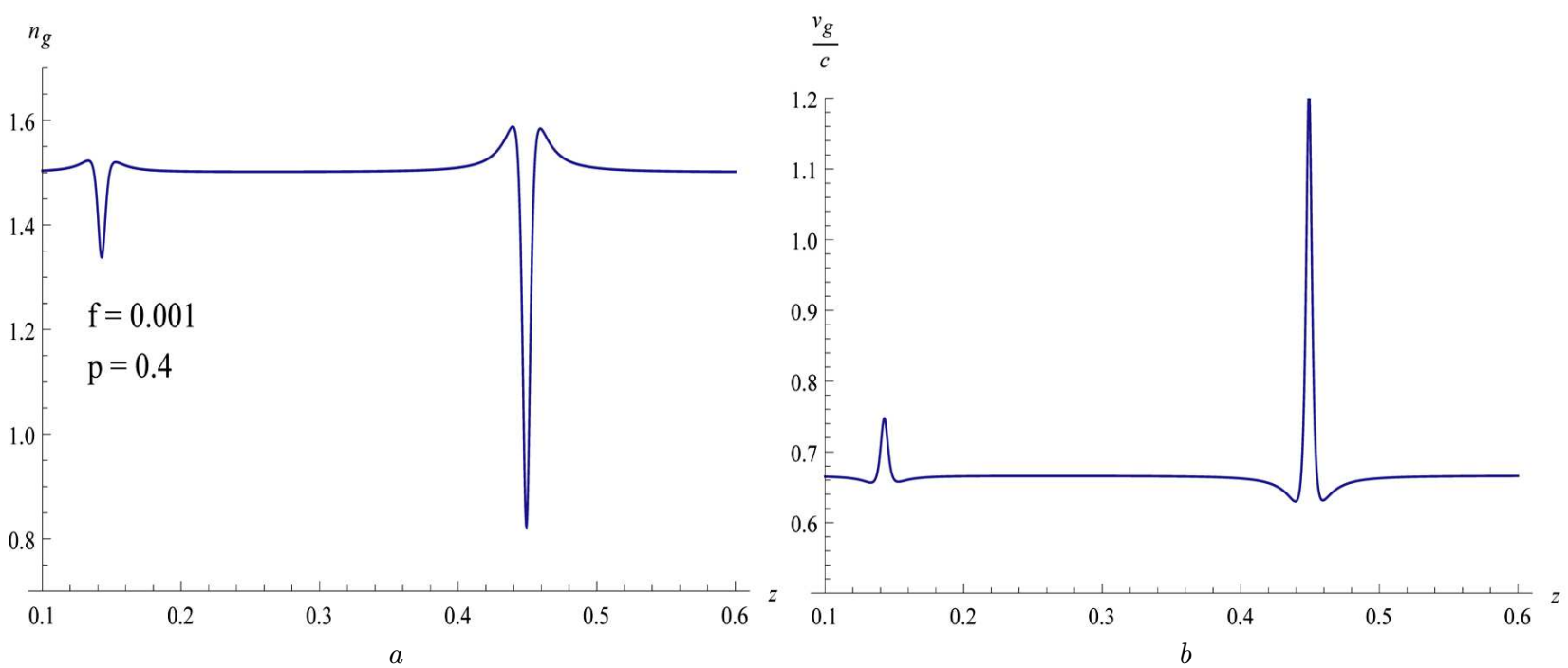

Fig. 7. Passive composite with tuned cylindrical nanoinclusions $\left(\epsilon_{h}^{\prime \prime}=0\right)$. Its group index $n_{g}$ versus the dimensionless frequency $z(a)$. The normalized group velocity $\mathbf{v}_{g} / c$ versus $z(b)$. Numerical values of composite parameters: metal fraction in the inclusion $p=0.4$, fraction of inclusions in the composite $f=0.001$
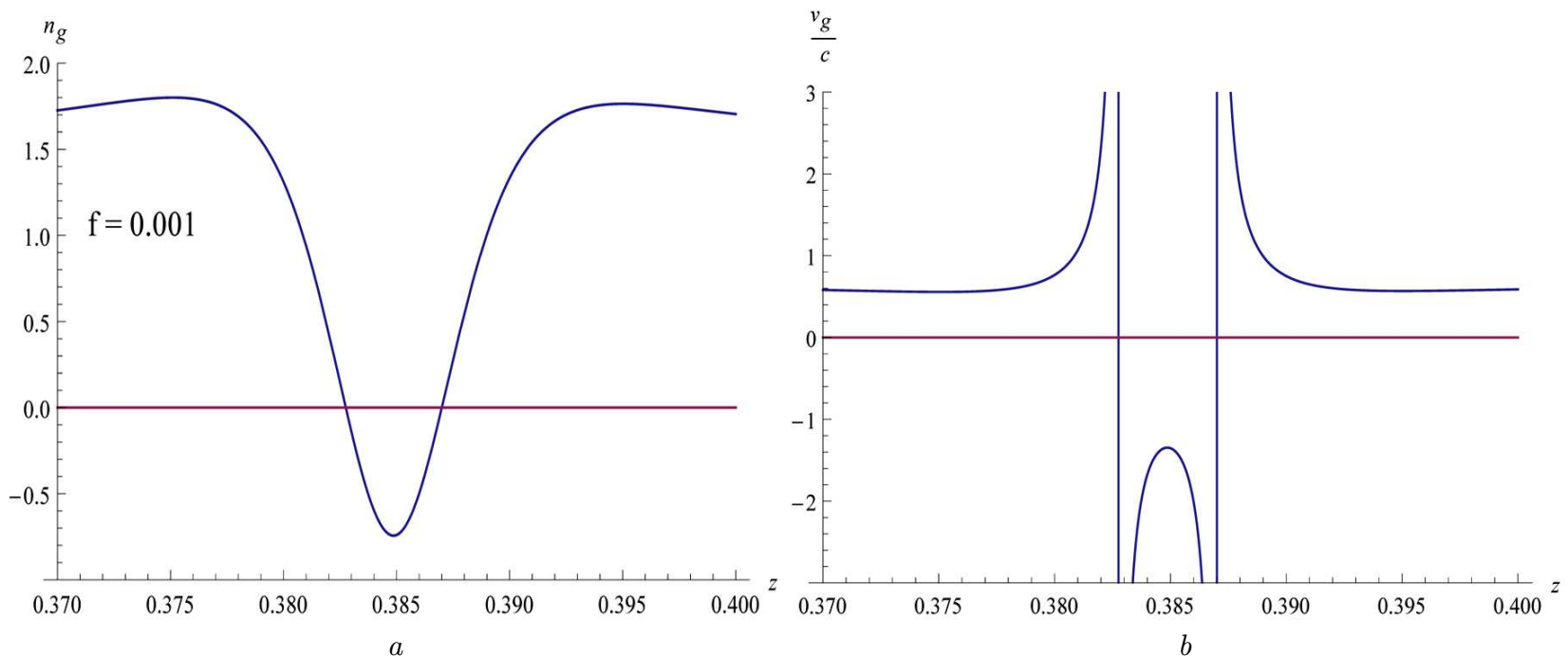

Fig. 8. Passive composite with pure metal inclusions $\left(\epsilon_{h}^{\prime \prime}=0\right)$. Its group index $n_{g}$ versus the dimensionless frequency $z$ ( $a$ ). The normalized group velocity $\mathbf{v}_{g} / c$ versus $z(b)$. Numerical values of composite parameters: fraction of inclusions in the composite $f=0.001$

Numerical results for the group index and group velocity for a composite of pure metal cylindrical inclusions in a passive host matrix are presented in Figs. 8 and 9 for $f=0.001$ and $f=0.003$, respectively. The curves for $\mathbf{v}_{g} / c$ show that, as the dimensionless frequency $z$ tends to the first asymptote from the left and to the second asymptote from the right, $\mathbf{v}_{g} / c \rightarrow+\infty$. But, between the two singular- ity points, slow backward light is obtained. The value $\mathbf{V}_{g} / c=-1.5$ for $f=0.001$ goes to $\mathbf{v}_{g} / c=-0.25$ for $f=0.003$.

\subsection{Active host matrix}

The numerical results for the group index and group velocity of a tuned inclusion in the active 

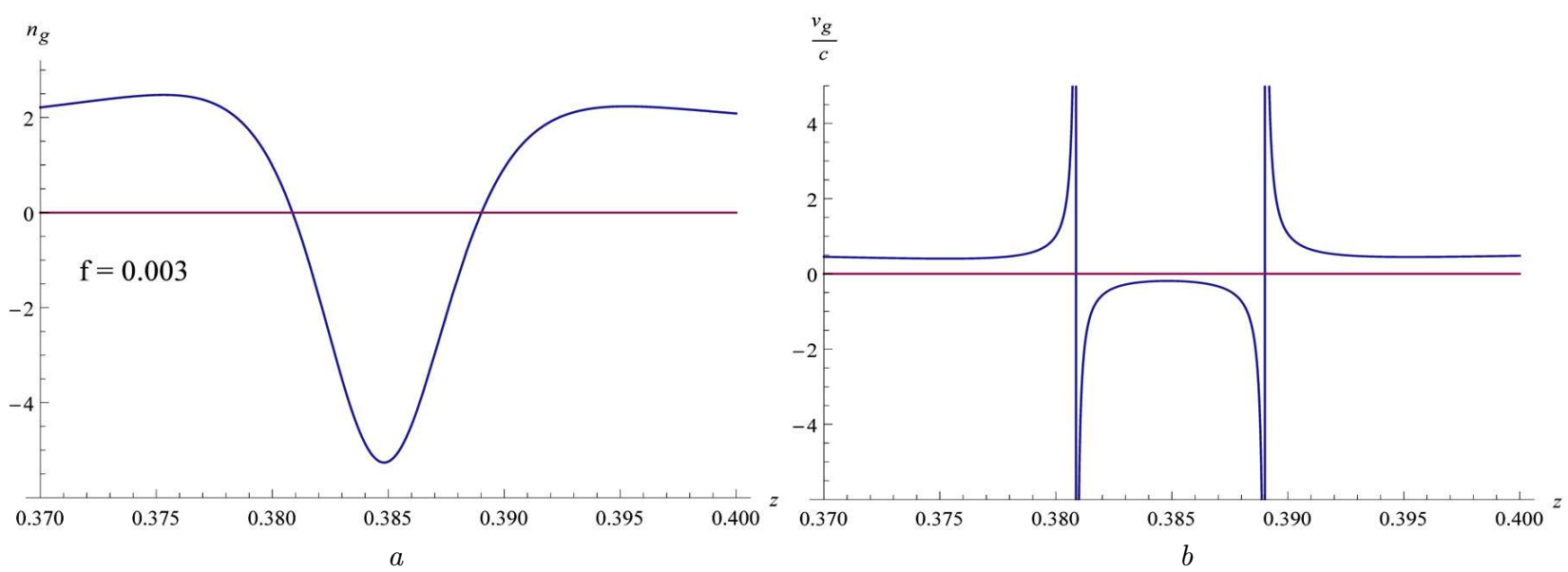

Fig. 9. Passive composite with pure metal inclusions $\left(\epsilon_{h}^{\prime \prime}=0\right)$. Its group index $n_{g}$ versus the dimensionless frequency $z(a)$. The normalized group velocity $v_{g} / c$ versus $z(b)$. Numerical values of composite parameters: fraction of inclusions in the composite $f=0.003$
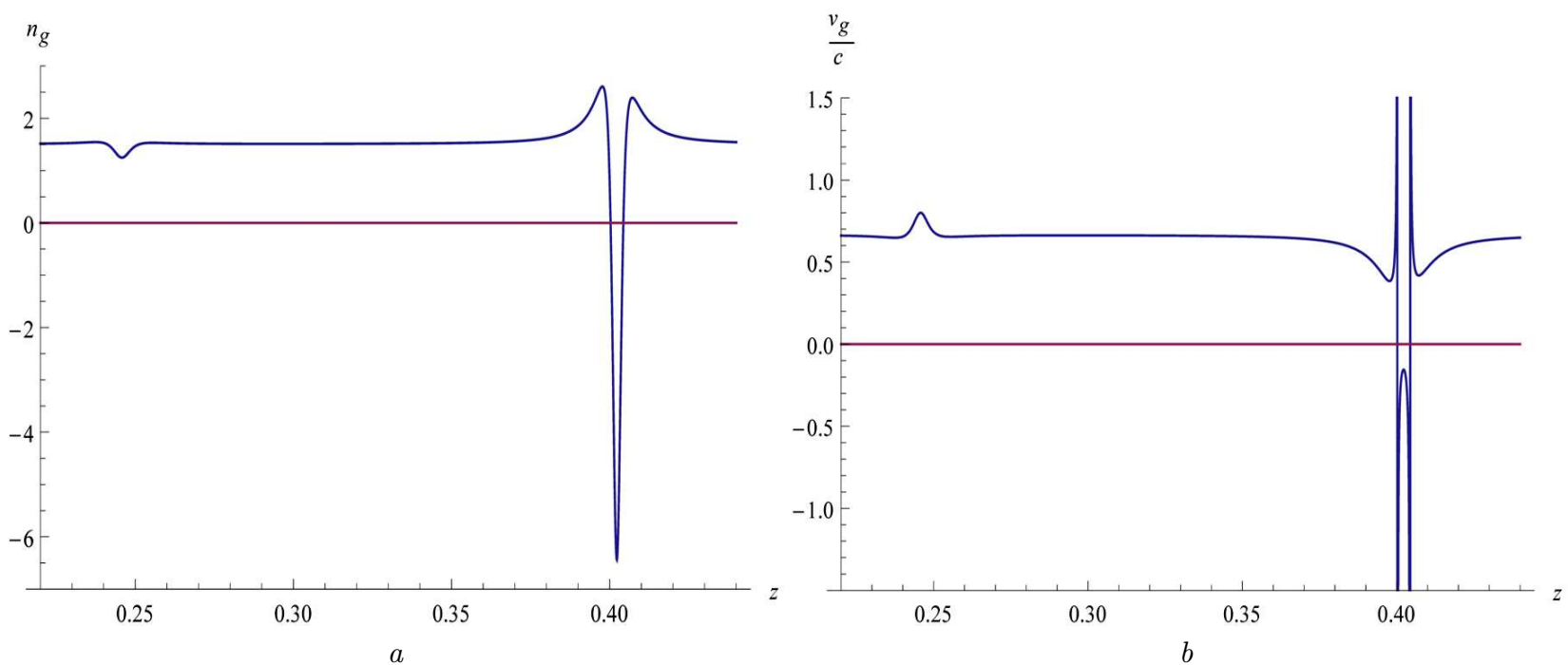

Fig. 10. Active composite with tuned cylindrical nanoinclusions $\left(\epsilon_{h}^{\prime \prime}=-0.13866\right)$. Its group index $n_{g}$ versus the dimensionless frequency $z(a)$. Yje normalized group velocity $\mathbf{v}_{g} / c$ versus $z(b)$. Numerical values of composite parameters: metal fraction in the inclusion $p=0.9$, fraction of inclusions in the composite $f=0.001$

host (i.e., $\epsilon_{h}^{\prime \prime}=-0.13866$ ) matrix are presented in Fig. 10, $a$ for $p=0.9$ with $f=0.001$. At the region of first resonant frequency, slow light with $\mathbf{v}_{g}=$ $=0.8 c$ is obtained. Near the second resonant frequency (Fig. 10, b), $\mathbf{v}_{g} / c$ has three branches. As $z$ goes to the first asymptote line from the left, and as $z$ tends to the second asymptote from the right, $\mathbf{v}_{g} / c \rightarrow+\infty$. Between the asymptotes, the negative slow light with $\mathbf{v}_{g}=-0.2 c$ is found.
Figures 11 and 12 present the group index and group velocity for the fractions of inclusions $f=$ 0.0001 and $f=0.0007$, respectively. For all fractions of inclusions under study, the group refractive index $n_{g}$ has single minimum. It becomes negative, as we increase $f$. For $f=0.0001$, we have got a superluminal group velocity $\mathbf{v}_{g}=4 c$ (see Fig. $11, b$ ). When we increase the value of $f$ to 0.0007 , the obtained curve demonstrates a completely different behavior, 


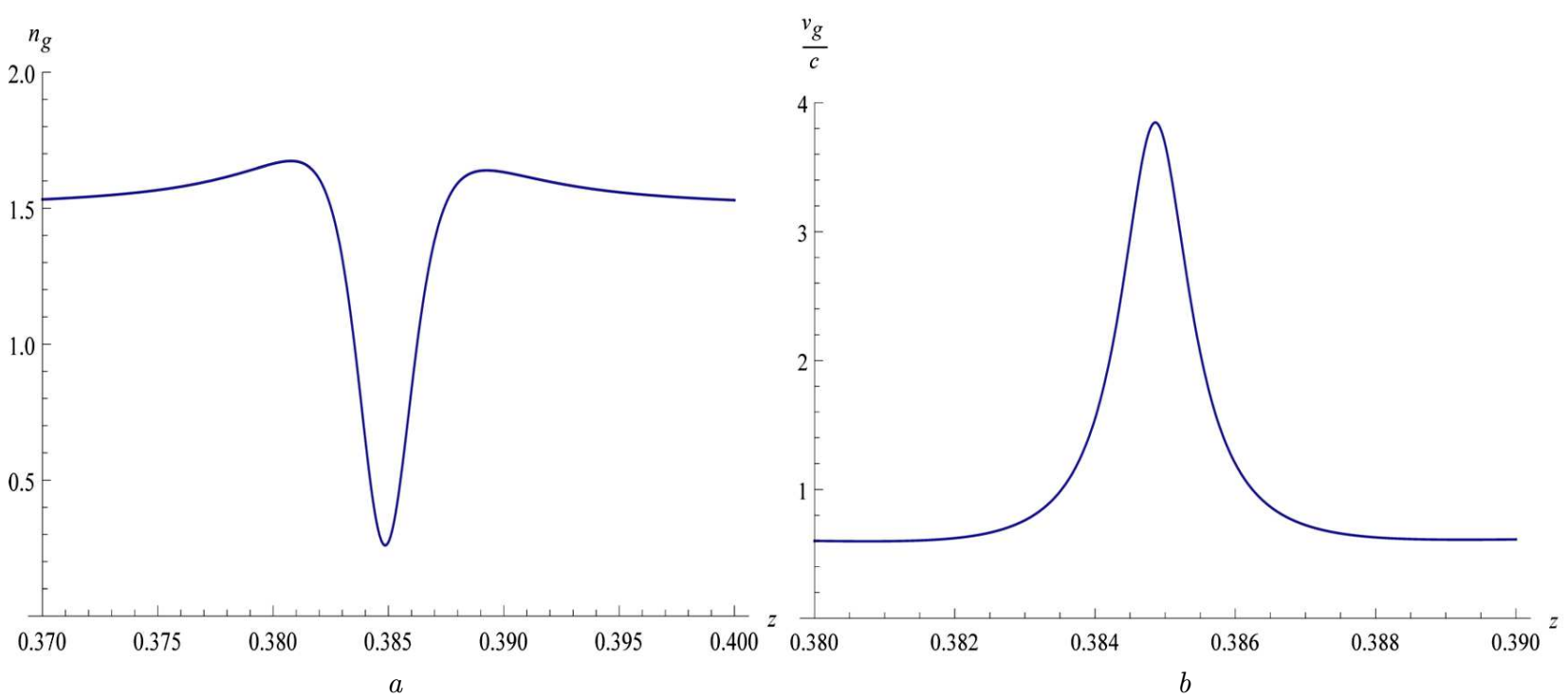

Fig. 11. Active composite with cylindrical pure metal inclusions $\left(\epsilon_{h}^{\prime \prime}=-0.115911\right)$. Its group index $n_{g}$ versus the dimensionless frequency $z(a)$. The normalized group velocity $\mathbf{v}_{g} / c$ versus $z(b)$. Numerical values of composite parameters: fraction of inclusions in the composite $f=0.0001$
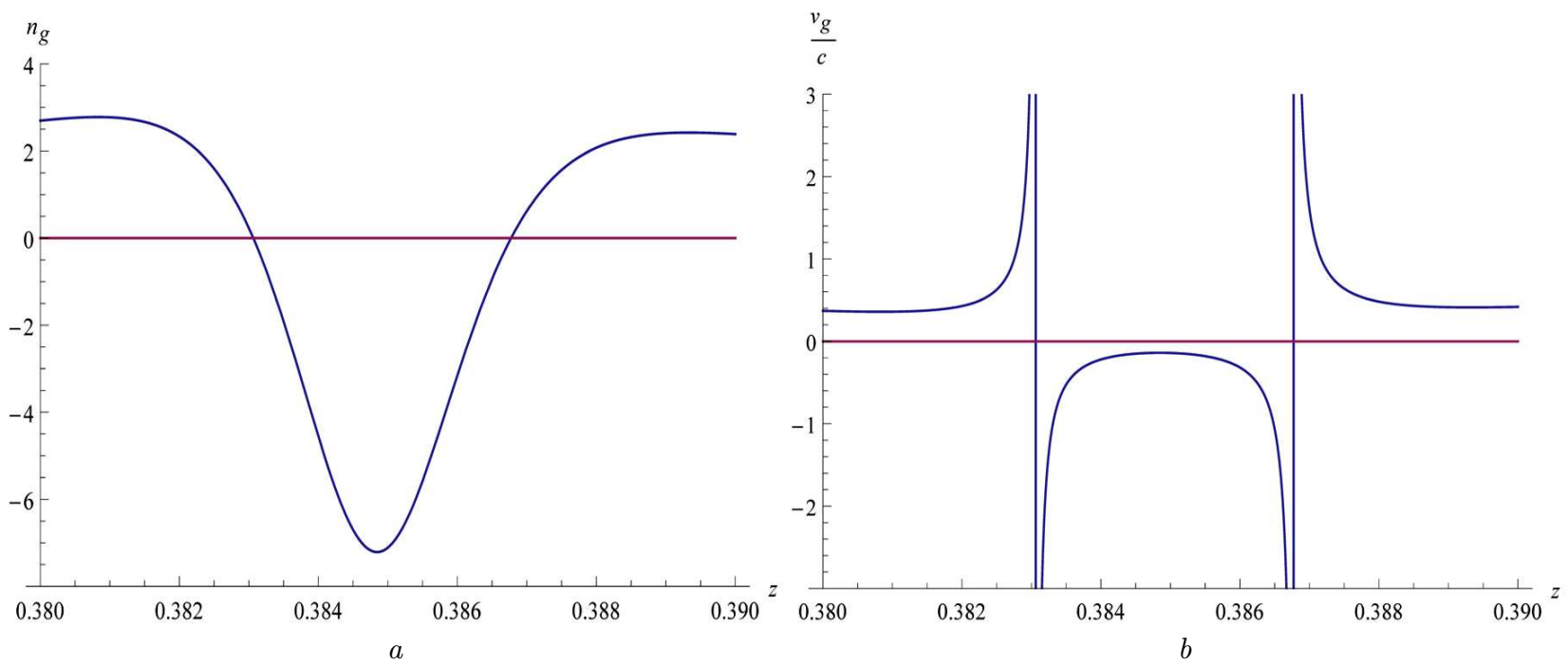

Fig. 12. Active composite with cylindrical pure metal inclusions $\left(\epsilon_{h}^{\prime \prime}=-0.115911\right)$. Its group index $n_{g}$ versus the dimensionless frequency $z(a)$. The normalized group velocity $v_{g} / c$ versus $z(b)$. Numerical values of composite parameters: fraction of inclusions in the composite $f=0.0007$

and we get three branches of the normalized group velocity. Far to the left from the first asymptote line and far from the right asymptotic line, the group velocity is low and is estimated as $\mathbf{v}_{g}=0.4 c$. As the frequency approaches the first asymptotic line from the left and the second asymptotic line from the right, ISSN 2071-0194. Ukr. J. Phys. 2021. Vol. 66, No. 4 $\mathbf{v}_{g} \rightarrow \infty$. Between the two singularity points, the negative slow group velocity of a light pulse is obtained.

\section{Conclusions}

The speed of light can be controlled in a composite of tuned (metal-covered dielectric) and pure metal 
cylindrical inclusions in passive and active host matrices. We have obtained that a light pulse can propagate with slow, superluminal, and even with negative group velocities in the stated composites. In both tuned and pure metal inclusions in a passive host matrix, the main challenge in the propagation distance of a light pulse is the strong absorption. By introducing a negative part in to the imaginary part of the permittivity, the problem of the strong attenuation of an electromagnetic wave by the composite can be considerably reduced. In a composite with coated inclusions, the metal fraction $p$ in inclusions plays the main role in varying the speed of a light/electromagnetic wave which propagates through it. But, for a composite with pure metal nanoinclusions, the fraction of inclusions $f$ affects the results related to the speed of waves. Since the speed of light can be controlled within the investigated model nanocompsoites, we can conclude these composites are a promising material for optical applications and further investigations.

I, Yilak Alemu Abbo, would like to express my deepest gratitude to my parents, sisters, and brothers for their support during my study at Addis Ababa University. It is my great pleasure to express my acknowledgement to my late instructor and advisor Prof. V.N. Mal'nev who passed unexpectedly; let his soul rest in peace.

1. R.W. Boyd. Slow and fast light: Fundamentals and applications. J. Modern Opt. 56, 1908 (2009).

2. L.V. Hau, S.E. Harris, Z. Dutton, C.H. Behroozi. Light speed reduction to 17 meters per second in ultra cold atomic gas. Nature 397, 594 (1999).

3. M.M. Kash, V.A. Sautenkov, A.S. Zibrov, L. Hollberg, E.S. Fry, M.O. Scully. Ultraslow group velocity and enhanced nonlinear optical effects in a coherently driven hot atomic gas Phys. Rev. Lett. 82, 5229 (1999).

4. A.M. Akulshin, S. Barreiro, A. Lezaman. Steep anomalous in coherently prepared $\mathrm{Rb}$ vapor. Phys. Rev. Lett. 83, 4277 (1999).

5. S.k. Kim, H.S. Moon, K.Kim, J.B.Kim. Observation of electromagnetically induced absorption in open systems regardless of angular momentum. Phys. Rev. A 68, 063813 (2003).

6. L.J. Wang, A. Kuzmich, A. Dogatiu. Gain assisted superluminal light propagation. Nature 406, 277 (2000).

7. A.M. Steinberg, P.G. Kwait, R.Y. Chiao. Measurement of the single-photon tunneling time. Phys. Rev. Lett. 71, 708 (1993).
8. M.S. Bigelow, N.N. Lepeshkin, R.W. Boyd. Ultra-slow and superluminal light propagation in solids at room temperature. J. Phys.: Condensed Matter. 16, 46 (2004).

9. V.N Mal'nev, S. Shewamare. Slow and fast light in metal/dielectric composite with passive and active host matrices. Physica B: Condensed Matter. 426, 52 (2013).

10. K.H. Kim, S.H. Choe. Slow and stooped light in active gain composite materials of metal nanoparticles: Ultralarge group index-bandwidth product predicted. Annalen der Physik 529, 8 (2017).

11. J.D. Jackson. Classical Electrodynamics (Wiley, 1999) [ISBN: 0-471-30932].

12. Y.A. Abbo, V.N. Mal'nev, A.A. Ismail. Local field enhancement at the core of cylindrical nanoinclusions embedded in a linear dielectric host matrix. Condens. Matter Phys. 19 (3), 33401 (2016).

13. M.M. Quinten. Optical Properties of Nanoparticle Systems, (Wiley-VCH, 2011) [ISBN-13: 978-3527410439].

14. S. Giordano. Effective medium theory for dispersions of dielectric ellipsoids. J. Electrostatics 58, 59 (2003).

15. R.D. Guenther. Modern Physics, (Wiley, 1990) [ISBN: 0.471-60538-7].

16. S. Shewamare, V.N. Mal'nev. Two optical bistability domains in composites of metal nanoparticles with nonlinear dielectric core. Phys. B: Condensed Matter. 407, 4837 (2012).

17. C.G.B. Garrett, D.E. MaCumber. Propagation of a Gaussian light pulse through an anomalous dispersion medium. Phys. Rev. A 1, 305 (1970).

18. S. Chu, S. Wong. Linear pulse propagation in an absorbing medium. Phys. Rev. Lett. 48, 738 (1982).

Received 13.04.20

\section{I. А. Аббо}

ПОВІЛЬНЕ І ШВИДКЕ СВІТЛО

У КОМПОЗИТІ МЕТАЛ/ДІЕЛЕКТРИК

З ЦИЛІНДРИЧНИМИ НАНОВКЛЮЧЕННЯМИ

В ПАСИВНИХ І АКТИВНИХ ЛІНІЙНИХ ДІЕЛЕКТРИЧНИХ МАТРИЦЯХ

У роботі представлені теоретичні дискусї та числові результати, отримані в результаті вивчення екстремальних значень швидкості світла в композиті метал/діелектрик, де циліндричні нановключення рівномірно розподілені в лінійній діелектричній матриці. Результати свідчать про те, що в області аномальної дисперсї̈, в рамках розглянутого нами підходу, світло може рухатися з груповою швидкістю більшою, ніж швидкість світла у вакуумі. У композиті з пасивною діелектричною матрицею світловий імпульс поглинається на дуже малій відстані. Проблему поглинання можна значно спростити, використовуючи активну матрицю.

Ключові слова: повільне світло, швидке світло, нанокомпозит, групова швидкість. 